\title{
The GP Score, a Simplified Formula (Bioptic Gleason Score Times Prostate Specific Antigen) as a Predictor for Biochemical Failure after Prostatectomy in Prostate Cancer
}

\author{
Norihito Soga ${ }^{a} \quad$ Yuji Ogura ${ }^{a} \quad$ Toshiaki Wakita $^{b} \quad$ Takumi Kageyama $^{c} \quad$ Jun Furusawa ${ }^{a}$ \\ aDepartment of Urology, Aichi Cancer Center Hospital, Nagoya; ${ }^{b}$ Department of Urology, Wakita Clinic, Aichi; \\ 'Department of Nephro-Urologic Surgery and Andrology, Mie University Graduate School of Medicine, Mie, Japan
}

\section{Key Words}

Predictive factors $\cdot$ Gleason score $\cdot$ Prostate cancer •

Prostate-specific antigen

\begin{abstract}
Objectives: We used a new GP score (Gleason score multiplied by prostate-specific antigen) without the T stage as a predictive value for biochemical failure (BCF) after prostatectomy. Materials and Methods: We assessed 459 prostate cancer patients who underwent prostatectomies at our institution. Three sub-groups were defined in terms of D'Amico classification risk (low, intermediate, and high) and Gleason score (low, < 50; intermediate, 50-100; and high GP score, $>100$ ). Risk factors for BCF were evaluated by multivariate analysis with a Cox hazard model. A log-rank test was used to compare the BCF rate in the 2 groups. Results: There was no significant difference in the non-BCF rate between the low risk and low GP score subgroups or the intermediate risk and intermediate GP score subgroups. In contrast, the non-BCF rate of the high GP score subgroup (42.1\%) was significantly lower than that of the high-risk subgroup $(66.1 \%$, log-rank $\mathrm{p}=0.008$ ). Based on multivariate analysis, a high GP score $(p=0.001$; HR 3.78; 95\%Cl 1.95-7.35) was a significant inde-
\end{abstract}

\section{KARGER}

Fax +4161306 1234

E-Mail karger@karger.com

www.karger.com
(C) 2019 The Author(s)

Published by S. Karger AG, Basel Open access

This article is licensed under the Creative Commons AttributionNonCommercial-NoDerivatives 4.0 International License (CC BYNonCommercial-NoDerivatives 4.0 International License (CC BYUsage and distribution for commercial purposes as well as any distribution of modified material requires written permission. pendent risk factor for BCF after prostatectomy. Conclusion: The GP score, consisting of two absolute numbers, may be a valuable predictive factor for BCF after prostatectomy, especially in the high-risk failure group. 02019 The Author(s)

Published by S. Karger AG, Basel

\section{Introduction}

In choosing a treatment strategy for prostate cancer, risk classification is necessary due to the heterogeneous nature of the disease. The major risk classification, named the D'Amico classification, consists of prostate-specific antigen (PSA) status, Gleason score (GS), and clinical $\mathrm{T}$ stage $[1,2]$. However, the clinical $\mathrm{T}$ stage, which is based on the digital examination and imaging estimation, could be a poor predictor for biochemical failure (BCF) after prostatectomy compared to other clinical factors, because of its inconsistency in predicting prognosis [3].

Another predictor, even though original Cancer of the Prostate Risk Assessment (CAPRA) was developed using preoperative parameters, including PSA, GS, T stage, percent positive biopsy and age [4], but CAPRA-S 
Table 1. Baseline characteristics

\begin{tabular}{lc}
\hline Clinical T stage & Values \\
\hline Number & 459 \\
Operation date & $2001 / 1-2013 / 12$ \\
Age, years & $64.9 \pm 5.4$ \\
PSA, ng/ml & $327(71.2 \%)$ \\
$0-10$ & $99(21.6 \%)$ \\
over 10-20 & $33(7.2 \%)$ \\
over 20 & \\
Gleason score & $249(54.2 \%)$ \\
$\leq 6$ & $153(33.4 \%)$ \\
= 7 & $57(12.4 \%)$ \\
$8-10$ & \\
Clinical T stage & $293(63.8 \%)$ \\
cT1c-T2a & $82(17.9 \%)$ \\
cT2b & $84(18.4 \%)$ \\
cT2c & \\
D'Amico classification & $135(29.4 \%)$ \\
Low & $190(41.4 \%)$ \\
Intermediate & $134(29.2 \%)$ \\
High & \\
GP score classification & $253(55.1 \%)$ \\
Low & $150(32.7 \%)$ \\
Intermediate & $56(12.2 \%)$ \\
High &
\end{tabular}

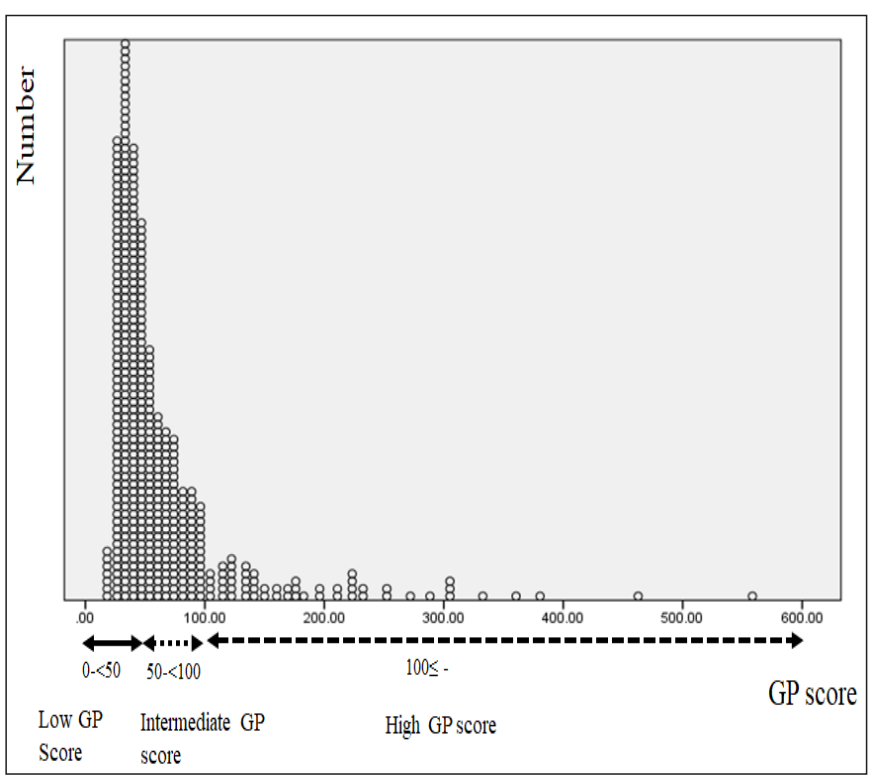

Fig. 1. Distribution of GP score.

score, consisted of PSA, surgical margins, seminal vesicle invasion, GS, extracapsular invasion and lymph node involvement. CAPRA-S has been used to predict recur-
Table 2. Comparison of D'Amico classification and GP score subgroup

\begin{tabular}{cllcc}
\hline & & \multicolumn{3}{c}{ GP score } \\
\cline { 3 - 5 } & Total & Low & Intermediate & High \\
\hline D'Amico classification & 459 & 253 & 150 & 56 \\
Low risk & 135 & $119(88.1 \%)$ & $16(11.9 \%)$ & $0(0 \%)$ \\
Intermediate risk & 190 & $90(47.4 \%)$ & $88(46.3 \%)$ & $12(6.3 \%)$ \\
High risk & 134 & $44(32.8 \%)$ & $46(34.4 \%)$ & $44(32.8 \%)$ \\
\hline
\end{tabular}

rence and mortality after prostatectomy [5]. However, since the score components can only be observed during surgery, the CAPRA-S is useful only after surgery.

Risk classifications used in clinical practice should be simple and easy to use. To address the limitations of the D'Amico classification and the CAPRA-S score, new and simpler predictive formulas $[6,7]$ and a scoring system [8] consisting of PSA and GS have been evaluated. To establish wider usage of these systems, additional improved formulas will be crucial.

In this study, we created a new predictive formula in which we multiplied the GS of a preoperative specimen by PSA, and named it the GP score. We assessed the effectiveness of this score as a preoperative predictive factor for PSA BCF after prostatectomy in prostate cancer, compared to classical D'Amico classification.

\section{Materials and Methods}

\section{Patient Population}

Between January 2001 and December 2013, we assessed 459 prostate cancer patients who underwent retropubic prostatectomies at our institution (table 1). Preoperatively, our central pathologist estimated GS based on the category in the 2005 International Society of Urological Pathology Consensus Conference on Gleason Grading of Prostatic Carcinoma [9]. This retrospective study was approved by the Institutional Review Board of our hospital.

\section{$P S A$ and $G S$}

Preoperative and postoperative PSA levels in serum were calculated based on the Tandem- $\mathrm{R}$ method (normal range $\leq 4.0 \mathrm{ng}$ / $\mathrm{ml}$ ), using the PSA calculation product of Abbott Japan (Chiba, Japan).

Preoperatively, an endorectal ultrasound guided prostatic biopsy was performed. The number of cores was sextant between January 2001 and October 2002; 8 cores were taken per biopsy, comprising sextant along with 2 cores of mid-line, between November 2002 and December 2006; and 10 cores were taken per biopsy, comprising sextant along with 4 cores of mid-line, after January 2007, respectively. To avoid interobserver variation, one central pathologist performed the evaluation GS for all bioptic samples. 




Fig. 2. Non-biochemical failure (BCF) rate in low-risk (blue line), intermediate-risk (red line) and high-risk (green line) subgroups, based on the D'Amico classification.

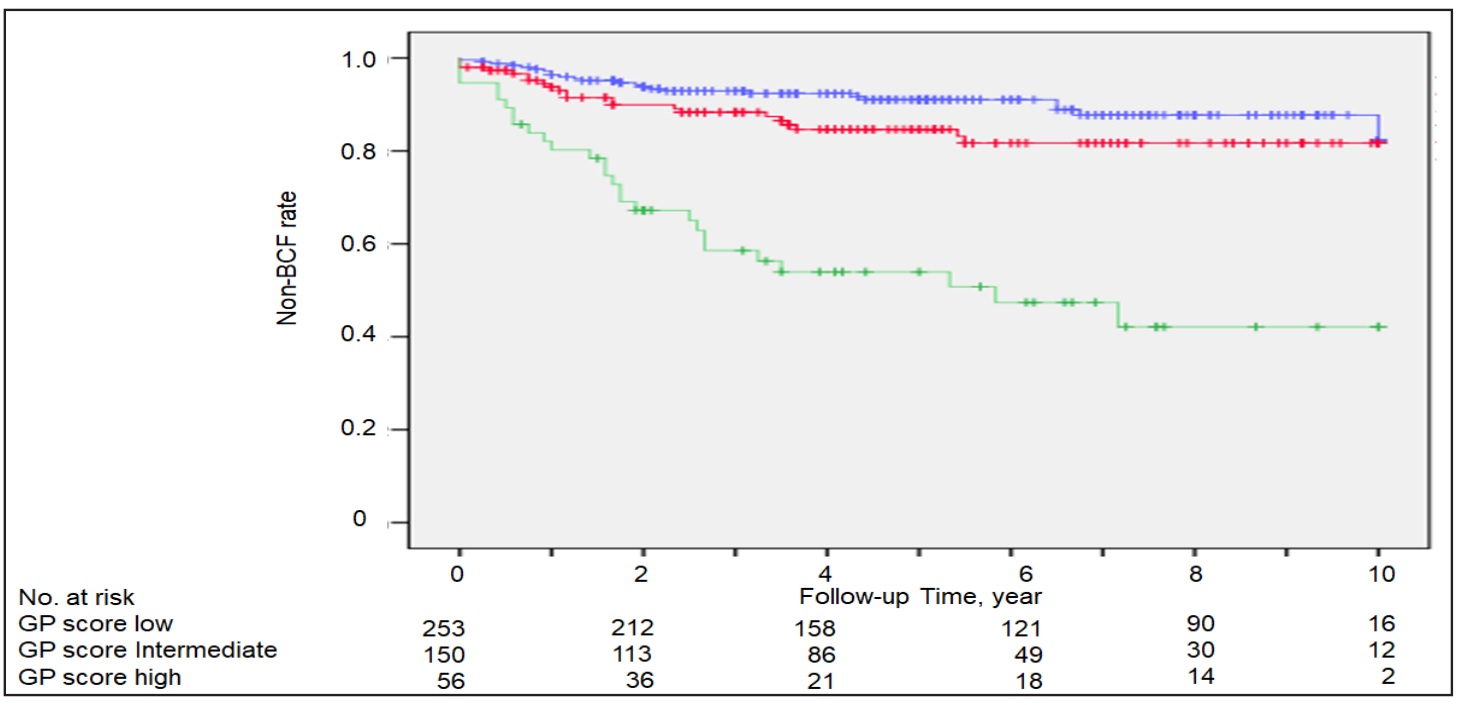

Fig. 3. Non-biochemical failure (BCF) rate in low GP score (blue line), intermediate GP score (red line) and high GP score (green line) subgroups, based on the GP score classification.

\section{D’Amico Classification}

Clinical risk was determined according to the D'Amico risk classification [1,2]. The numbers of patients in the low-, intermediate- and high-risk subgroups were 135 (29.4\%), $190(41.4 \%)$ and $134(29.2 \%)$, respectively.

\section{GP Score}

We named the new formula the GP score: specifically, bioptic GS multiplied by PSA. Figure 1 shows the distribution of the cases. GP scores ranged from 15.6 to 558, and the mean and standard deviation were 66.0 and 60.7 , respectively. With the 3 peaks 
Table 3. Multivariate analysis (Cox proportional hazards model) of predictors for BCF after prostatectomy

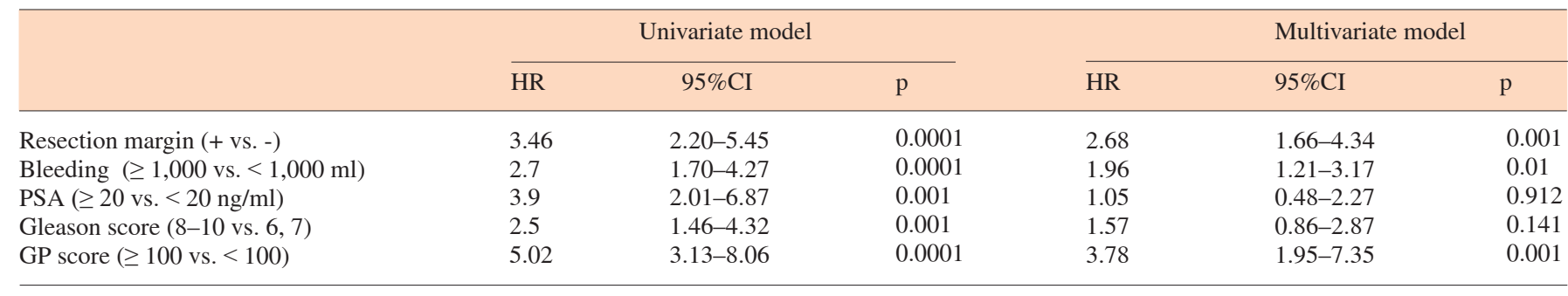

of distribution estimated in figure 1 , we classified the cases into 3 subgroups: low, < 50; intermediate, from 50 to 100; and high, > 100. The numbers of patients in the subgroups were $253(55.1 \%)$, $150(32.7 \%)$ and $56(12.2 \%)$, respectively.

\section{Definition of Progression}

The definition of BCF of PSA was the first day with a serum PSA level exceeding $0.2 \mathrm{ng} / \mathrm{ml}$ followed by a 3-point elevation. If the PSA nadir after prostatectomy did not go below $0.2 \mathrm{ng} / \mathrm{ml}$, the date of surgery was the date of PSA BCF. The period between the date of surgery and the PSA BCF date was evaluated.

\section{Statistical Analysis}

The significance of the difference in the distribution of cases was estimated by a chi-squared test. A multivariate analysis with a Cox hazard model was performed to identify relevant prognostic factors for BCF after prostatectomy for prostate cancer. A logrank test was used to compare the BCF rates in the D'Amico and the GP groups. Differences with p values of less than 0.05 were considered statistically significant. All statistical analyses were performed with SPSS version 15 software (SPSS, Chicago, IL, USA).

\section{Results}

The distributions of cases depending on the D'Amico and the GP score were determined. The matching ratio between the D'Amico classification and the GP score subgroups was $88.1 \%$ in the low, $46.3 \%$ in the intermediate, and $32.8 \%$ in the high subgroups, respectively. The matching ratio decreased significantly $(\mathrm{p}<0.001)$, and was associated with elevated risk value (table 2).

During a median follow-up period of 56.5 months, the 10 -year non-BCF rates were $90.4,79.8$, and $66.1 \%$ for the low-, intermediate-, and high-risk subgroups, respectively (fig. 2). For the low, intermediate, and high GP score subgroups, the 10-year non-BCF rates were 85.7, 81.6 , and $42.1 \%$, respectively (fig. 3 ). There were no significant differences in the non-BCF rates between the low risk and low GP subgroups or the intermediate risk and intermediate GP subgroups (fig. 2a, b). In contrast, the non-BCF rate of the high GP subgroup was significantly lower than that of the high-risk subgroup (42.1 vs. $66.1 \%, \log$-rank $\mathrm{p}=0.008$ ).

Based on the univariate model, positive resection margin, bleeding over $1,000 \mathrm{ml}, \mathrm{PSA} \geq 20 \mathrm{ng} / \mathrm{ml}, \mathrm{GS} \geq$ 8 , and GP score $\geq 100$ (high GP score) were significant risk factors for $\mathrm{BCF}$ after prostatectomy. In multivariate analysis, a high GP score ( $\mathrm{p}=0.001$; HR 3.78 ; 95\%CI 1.95-7.35) was a significant independent risk factor for $\mathrm{BCF}$ after prostatectomy, associated with positive resection margin $(\mathrm{p}=0.001$; HR 2.68; 95\%CI 1.66-4.34) and bleeding over $1,000 \mathrm{ml}(\mathrm{p}=0.01 ; \mathrm{HR} 1.96 ; 95 \% \mathrm{CI}$ 1.21-3.17) (table 3).

\section{Discussion}

Choosing the appropriate management of prostate cancer can be complicated due to the heterogeneity of prostate cancer itself and the availability of several treatment options. Even though prostatectomy is generally considered the optimal option, patients often want to know the probability of post-treatment $\mathrm{BCF}$ in preoperative stage. We looked for a simple formula to provide a useful preoperative predictor for BCF.

We created and assessed the GP score, which takes the GS and PSA but not the clinical T stage into account. According to this evaluation, the low GP subgroup and the D'Amico low risk subgroup had similar rates of recurrence, as did the patients with intermediate GP compared with those with D'Amico intermediate risk. Surprisingly, the high GP subgroup (42.1\%) had a lower rate of non -BCF compared with the D'Amico high-risk subgroup $(66.1 \%)$. 
Various formulas that include the GS and PSA with or without the T stage have been reported upon as preoperative predictors for BCF or lymph node metastasis. The Roach formula, $2 / 3 \times$ PSA $+(G S-6) \times 10$, was evaluated for predicting the risk of lymph node involvement in localized prostate cancer [6] , and it predicted lymph node metastasis in extended pelvic lymph node cases in prostate cancer after prostatectomy [10] . However, other groups have reported that the Roach formula overestimated pelvic lymph node risk [7]. The Yu formula includes GS, PSA and T stage. Yu et al. [11] reported that this formula $(\mathrm{GS}-5) \times[\mathrm{PSA} / 3+(1.5 \times \mathrm{T})]$, where $\mathrm{T}=0$, 1 , and 2 of clinical stage T1c, T2a, and T2b/T2c, respectively), was a useful predictor of high risk of lymph node metastasis after prostatectomy. Still another scoring system, named PRIX (prostate risk index), consists of PSA, GS, and T stage. PRIX was a crucial predictor of lymph node metastasis according to a Partin cancer-staging nomogram [12]. BCF after prostatectomy for prostate cancer [8], and high-risk patient selection for high-dose-rate interstitial brachytherapy [13] were respectively.

Compared to these formulas, our GP score formula may be simpler and easier to calculate, and may prove to be a useful tool in clinical practice for treatment decision-making before surgery for prostate cancer.

Can the clinical $\mathrm{T}$ stage contribute to improvements in predictive value? Recently, multiparametric magnetic resonance imaging was found to be useful in T staging, especially for extracapsular invasion [14]. In addition, a positive computed tomography result was associated with PSA, GS, and T stage [15]. In the future, after improvements in radiological technology improve the accuracy of clinical $\mathrm{T}$ stage identification, a new formula including the clinical $\mathrm{T}$ stage can be considered.

In terms of scoring based on PSA and GS, we must treat PSA and GS differently. As PSA is a numerical value, it can be easily used in scoring. However, GS is a more subjective value, as it is affected by the pathologist's judgment. GS is a unique grading system specifically used for prostate cancer [15], and $44-48 \%$ of cases may be graded differently by different pathologists $[17,18]$. In fact, according to our previous paper, only $59.5 \%$ of cases were given concordant GS by local and central pathologists, which affected the distribution risk classification [19]. Thus, just as in this report, the central pathologists' GS will be indispensable in calculating the GP score, to avoid validations.

What types of high-risk cases could be defined by a high GP score? According to our data, the non-BCF rate of the high GP subgroup was significantly lower than that of the D'Amico classification high-risk subgroup (42.1 vs. $66.1 \%, \log$-rank $p=0.008)$. The matching ratio between the high GP and high-risk group was only $32.8 \%$, which indicates that the high GP subgroup patients had different characteristics compared with the high-risk group. In addition, high GP was a significant independent risk factor for BCF after prostatectomy, associated with a positive resection margin. In general, since positive surgical margin should be a strong predictor for $\mathrm{BCF}$ after prostatectomy [20], high GP included the high risk cases with other risk factors, except for positive surgical margins. The one candidate risk factor will be micro metastases in the lymph nodes or other organs.

This study has several limitations. First, this study had a retrospective design and a low number of cases. Second, more than $50 \%$ of our patients undergoing prostatectomy had a GS of 6; their background was quite different from present practice, with more and more highrisk cases undergoing surgery. Third, since the number of cores obtained by endorectal ultrasound guided prostatic biopsy changed with the time period of the biopsy, the preoperative GS may have been affected with time. Fourth, between 2001 and 2013, medical practices have changed, which would have affected the treatment strategy and clinical results in our study.

However, according to our results, the GP score should be useful as a predictive value for PSA BCF after prostatectomy. In addition, the GP score has the advantages of simplicity in using two commonly used factors, GS and PSA. Therefore, this formula may contribute to prostate cancer treatment decision-making in clinical practice. For the future, the efficacy of GP score should be evaluated on the larger cohorts. 


\section{References}

1 D'Amico AV, Whittington R, Malkowicz SB, Schultz D, Blank K, Broderick GA, Tomaszewski JE, Renshaw AA, Kaplan I, Beard CJ, Wein A: Biochemical outcome after radical prostatectomy, external beam radiation therapy, or interstitial radiation therapy for clinically localized prostate cancer. JAMA 1998; 280:969-974.

-2 D'Amico AV, Whittington R, Malkowicz SB, Cote K, Loffredo M, Schultz D, Chen MH, Tomaszewski JE, Renshaw AA, Wein A, Richie JP: Biochemical outcome after radical prostatectomy or external beam radiation therapy for patients with clinically localized prostate carcinoma in the prostate specific antigen era. Cancer 2002;95:281-286.

-3 Reese AC, Cooperberg MR, Carroll PR: Minimal impact of clinical stage on prostate cancer prognosis among contemporary patients with clinically localized disease. J Urol 2010; 184:114-119.

4 Cooperberg MR. Pasta DJ, Elkin EP, Litwin MS, Latini DM, Du Chane J, Carroll PR: The University of California, San Francisco Cancer of the Prostate Risk Assessment score: a straightforward and reliable preoperative predictor of disease recurrence after radical prostatectomy. J Urol 2005;173:1938-1942.

-5 Punnen S, Freedland SJ, Presti JC Jr, Aronson WJ, Terris MK, Kane CJ, Amling CL, Carroll PR, Cooperberg MR: Multi-institutional validation of the CAPRA-S score to predict disease recurrence and mortality after radical prostatectomy. Eur Urol 2014;65:1171-1177.

-6 Roach M 3rd, Marquez C, Yuo HS, Narayan P, Coleman L, Nseyo UO, Navvab Z, Carroll PR: Predicting the risk of lymph node involvement using the pre-treatment prostate specific antigen and Gleason score in men with clinically localized prostate cancer. Int J Radiat Oncol Biol Phys 1994;28:33-37.
7 Nguyen PL, Chen MH, Hoffman KE, Katz MS, D'Amico AV: Predicting the risk of pelvic node involvement among men with prostate cancer in the contemporary era. Int J Radiat Oncol Biol Phys 2009;74:104-109.

8 Yoshida T, Nakayama M, Matsuzaki K, Kobayashi Y, Takeda K, Arai Y, Kakimoto K, Nishimura K: Validation of the Prostate Cancer Risk Index (PRIX): a simple scoring system to predict risk of biochemical relapse after radical prostatectomy for prostate cancer. Jpn J Clin Oncol 2011;41:1271-1276.

-9 Epstein JI, Allsbrook WC Jr, Amin MB, Egevad LL: The 2005 International Society of Urological Pathology (ISUP) Consensus Conference on Gleason grading of prostatic carcinoma. Am J Surg Pathol 2005;29:1228-1242.

10 Abdollah F, Cozzarini C, Sun M, Suardi N, Gallina A, Passoni NM, Bianchi M, Tutolo M, Fossati N, Nini A, Dell'oglio P, Salonia A, Karakiewicz P, Montorsi F, Briganti A: Assessing the most accurate formula to predict the risk of lymph node metastases from prostate cancer in contemporary patients treated with radical prostatectomy and extended pelvic lymph node dissection. Radiother Oncol 2013;109:211-216.

11 Yu JB, Makarov DV, Gross C: A new formula for prostate cancer lymph node risk. Int J Radiat Oncol Biol Phys 2011;80:69-75.

12 Yoshioka Y, Inoue T: Prostate Risk Index (PRIX) as a new method of risk classification for clinically localized prostate cancer. Strahlenther Onkol 2007;183:490-496.

13 Yoshida K, Yamazaki H, Nakamura S, Masui K, Kotsuma T, Akiyama H, Tanaka E, Yoshioka Y: Role of novel risk classification method, Prostate Cancer Risk Index (PRIX) for clinically localized prostate cancer after highdose-rate interstitial brachytherapy as monotherapy. Anticancer Res 2014;34:3077-3081.
14 Somford DM, Hamoen EH, Futterer JJ, van Basten JP, Hulsbergen-van de Kaa CA, Vreuls W, van Oort IM, Vergunst H, Kiemeney LA, Barentsz JO, Witjes JA: The predictive value of endorectal 3 Tesla multiparametric magnetic resonance imaging for extraprostatic extension in patients with low, intermediate and high risk prostate cancer. J Urol 2013;190:1728-1734.

15 Risko R, Merdan S, Womble PR, Barnett C, Ye Z, Linsell SM, Montie JE, Miller DC, Denton BT: Clinical predictors and recommendations for staging computed tomography scan among men with prostate cancer. Urology 2014;84:1329-1334.

16 Gleason DF: Classification of prostatic carcinomas. Cancer Chemother Rep 1966;50:125128.

17 Sooriakumaran P, Lovell DP, Henderson A, Denham P, Langley SE, Laing RW: Gleason scoring varies among pathologists and this affects clinical risk in patients with prostate cancer. Clin Oncol (R Coll Radiol) 2005; 17:655-658.

Nguyen PL, Schultz D, Renshaw AA, Vol-

18 lmer RT, Welch WR, Cote K, D'Amico AV: The impact of pathology review on treatment recommendations for patients with adenocarcinoma of the prostate. Urol Oncol 2004; 22:295-299.

Soga N, Yatabe Y, Kageyama T, Ogura

19 Y, Hayashi N: Review of bioptic Gleason scores by central pathologist modifies the risk classification in prostate cancer. Urol Int 2015;95:452-456.

Blute ML, Bostwick DG, Bergstralh EJ, Sle-

20 zak JM, Martin SK, Amling CL, Zincke H: Anatomic site-specific positive margins in organ-confined prostate cancer and its impact on outcome after radical prostatectomy. Urology 1997;50:733-739. 